\title{
Imaging-based molecular barcoding with pixelated dielectric metasurfaces
}

\author{
Andreas Tittl ${ }^{1}$, Aleksandrs Leitis ${ }^{1}$, Mingkai Liu ${ }^{2}$, Filiz Yesilkoy ${ }^{1}$, Duk-Yong Choi ${ }^{3}$, \\ Dragomir N. Neshev ${ }^{2}$, Yuri S. Kivshar ${ }^{2}$, and Hatice Altug ${ }^{1 *}$ \\ ${ }^{1}$ Institute of BioEngineering, École Polytechnique Fédérale de Lausanne (EPFL), \\ Lausanne 1015, Switzerland \\ ${ }^{2}$ Nonlinear Physics Centre, Research School of Physics and Engineering, \\ Australian National University, Canberra, ACT 2601, Australia. \\ ${ }^{3}$ Laser Physics Centre, Research School of Physics and Engineering, \\ Australian National University, Canberra, ACT 2601, Australia. \\ *Correspondence to: hatice.altug@epfl.ch
}

\begin{abstract}
:
Metasurfaces offer unique opportunities for wavefront control, advanced light focusing, and flat optics. Here, we introduce an imaging-based nanophotonic method for detecting mid-infrared molecular fingerprints, and implement it for the chemical identification and compositional analysis of surface-adsorbed analytes. Our technique leverages a two-dimensional pixelated dielectric metasurface featuring a range of ultra-sharp resonances each tuned to discrete frequencies, enabling us to read out molecular absorption signatures at multiple spectral points and to translate this information into a barcode-like spatial absorption map for imaging. We successfully detect the signatures of biological, polymer, and pesticide molecules with high sensitivity, covering applications ranging from biosensing to environmental monitoring. Significantly, our chemically specific technique is capable of resolving absorption fingerprints without the need for spectrometry, frequency scanning, or moving mechanical parts, redefining the boundaries of surface-enhanced molecular detection and paving the way towards chipintegrated mid-infrared spectroscopy.
\end{abstract}

\section{One Sentence Summary:}

Pixelated dielectric metasurfaces enable imaging-based detection of molecular fingerprints for integrated mid-infrared biosensing and environmental monitoring.

\section{Main text:}

The mid-infrared (mid-IR) spectral range is essential for sensing due to the presence of characteristic molecular absorption fingerprints originating from the intrinsic vibrational modes of chemical bonds. Mid-IR spectroscopy is widely recognized as the gold standard for chemical analysis, since it allows a direct characterization of molecular structures with chemical specificity unique to this spectral range (1). It is also a powerful nondestructive and label-free technique for identifying biochemical building blocks, including proteins, lipids, and DNA, among others. However, due to the severe mismatch between mid-IR wavelengths and 
dimensions of molecules, the sensitivity of mid-IR spectroscopy is limited when detecting signals from nanometer-scale volumes (2), biological membranes (3), or low numbers of surfaceadsorbed molecules (4).

Advances in nanophotonics have revealed a promising paradigm to overcome this limitation by exploiting the strong near-field enhancement of subwavelength resonators. When the resonance is spectrally overlapped with the absorption fingerprints, the enhanced moleculeresonator coupling can lead to a change in either the frequency or the strength of the resonance, from which the molecular fingerprints can be extracted. This concept, dubbed surface-enhanced infrared absorption (SEIRA), has been realized using various plasmonic platforms (5-7); however, the actual achieved performance is still far from ideal due to the inherent limitation of low quality factors (Q-factors) imposed by Ohmic loss.

Recently, nanostructured resonators based on high-index dielectric materials have emerged as essential building blocks for various metadevices due to their low intrinsic loss and CMOS compatibility, demonstrating unique capabilities for controlling the propagation and localization of light $(8,9)$. Many exciting applications including generalized wave front control $(10,11)$, ultra-thin optical elements $(12,13)$, and antennas for nanoscale light concentration (14) have been shown experimentally. A key concept underlying the specific functionalities of many metasurface approaches is their use of constituent elements with spatially varying optical properties. However, so far, the potential of metasurface-based SEIRA with both spectral and spatial control over the nanoscale field enhancement has not been realized.

In this letter, we report a mid-IR nanophotonic sensor based on all-dielectric high-Q metasurface elements and demonstrate its capabilities for enhancing, detecting, and differentiating the absorption fingerprints of various molecules. In contrast to previous approaches where high-Q resonances in metasurfaces are generated via the interference of superradiant and sub-radiant modes (15-17), our design exploits the collective behavior of Mie resonances, which allows strong near-field enhancement and a spectrally "clean" high-Q resonance that can be excited without additional resonance background. This property is particularly attractive since it allows for the highly spectrally selective enhancement of molecular fingerprint information. Specifically, we implement a 2D array of high-Q metasurface pixels, where the resonance positions of individual metapixels are linearly varied over a target mid-IR fingerprint range. This configuration allows us to assign each resonance position to a specific pixel of the metasurface, establishing a one-to-one mapping between spectral and spatial information (Fig. 1A). By comparing the imaging-based readout of this spatially encoded vibrational information before and after the coating of target analyte molecules, we experimentally demonstrate that our method can deliver chemically specific molecular barcodes suitable for highly integrated chemical identification and compositional analysis.

Individual metapixels contain a zig-zag array of anisotropic resonators composed of hydrogenated amorphous silicon (a-Si:H), which provide ultra-sharp resonances when excited with linearly polarized light and allow for straightforward resonance tuning via scaling of its geometrical parameters by a factor S (Fig. 1B). A detailed analysis of the collective resonance concept is given in Supplementary Section 1. Numerically simulated reflectance spectra of an exemplary 5 x 5 metasurface pixel array with a scaling factor variation from $S=1.0$ to $S=1.3$ show pronounced resonance peaks with near-unity reflectance intensity, high resonance sharpness (average $Q>200$ ), and linear tunability of the resonance positions over a spectral coverage range from $1350 \mathrm{~cm}^{-1}$ to $1750 \mathrm{~cm}^{-1}$ (Fig. 1C). Our metapixel design also provides more 
than three orders of magnitude enhancement of the local electric near-field intensity confined to the resonator surface, making it ideal for amplifying and detecting the molecular vibrations of adsorbed molecules (Fig. 1D, Fig. S1).

The target spectral range from $1350 \mathrm{~cm}^{-1}$ to $1750 \mathrm{~cm}^{-1}$ contains a multitude of characteristic molecular stretching/bending vibrations found in hydrocarbons and amino acids, making it crucial for detecting and differentiating the absorption signatures of biomolecules, environmental pollutants, and polymeric species, among others. We first focus on a biosensing application by showing chemical-specific protein detection. The distinct protein absorption fingerprint is governed by the amide I and II vibrational bands located at around $1660 \mathrm{~cm}^{-1}$ and $1550 \mathrm{~cm}^{-1}$, respectively.

A sub-5 nm conformal protein layer, modeled to cover the pixelated metasurface, causes a pronounced modulation of the individual metapixel reflectance spectra due to the coupling between the molecular vibrations and the enhanced electric near-fields around the dielectric resonators. This reflectance modulation manifests primarily as an attenuation and broadening of the metapixel resonance, which are correlated with the strength of the amide I and II molecular vibrations (Fig. 1E). Significantly, the envelope of the metapixel reflectance spectra unambiguously reproduces the protein absorption signature, confirming that our pixelated metasurface concept can perform efficient molecular fingerprint detection.

Individual metapixel resonances provide linewidths (full width at half maximum, FWHM) of around $7.5 \mathrm{~cm}^{-1}$, which are much narrower than the spectral feature size of the individual amide I and II absorption bands at around $60 \mathrm{~cm}^{-1}$. This is in strong contrast to metal-based antennas used in plasmonic SEIRA approaches, which typically exhibit linewidths above $200 \mathrm{~cm}^{-1}$ limited by the intrinsic damping of the metal (5). This conceptual advantage allows us to read out the protein absorption signature at multiple discrete frequency points and to translate this spectrally resolved absorption information into a barcode-like spatial map of the individual metapixel absorption signals (Fig. 1F). 


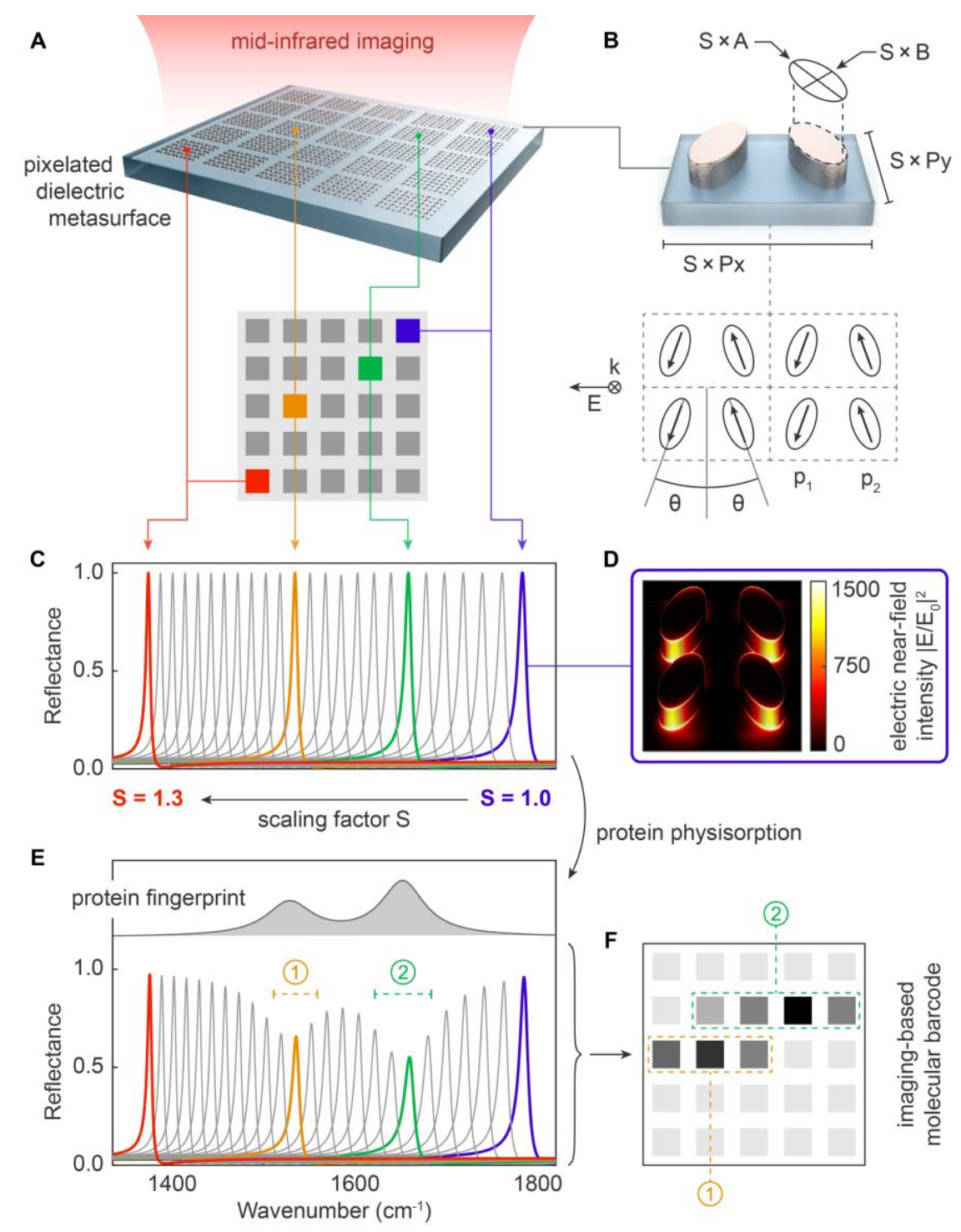

Fig. 1: Molecular fingerprint detection with pixelated dielectric metasurfaces. (A) Pixelated metasurface composed of a two-dimensional array of high-Q resonant metapixels with resonance frequencies tuned over a target molecular fingerprint range. (B) Spectrally clean high-Q > 200 resonances are provided by zig-zag arrays of anisotropic resonators composed of hydrogenated amorphous silicon (a-Si:H). Resonance frequencies are controlled by scaling the unit cell lateral dimensions by a factor $S$. (C) Numerically simulated metapixel reflectance spectra for different values of the scaling parameter $S$, chosen to cover the amide band spectral region around 1600 $\mathrm{cm}^{-1}$. Geometrical parameters are $\mathrm{A}=1.96 \mu \mathrm{m}, \mathrm{B}=0.96 \mu \mathrm{m}, \mathrm{Px}=3.92 \mu \mathrm{m}$, and $\mathrm{Py}=2.26 \mu \mathrm{m}$, with a fixed structure height of $\mathrm{H}=0.7 \mu \mathrm{m}$ and an orientation angle of $\theta=20^{\circ}$. (D) Simulated enhancement of electric near-field intensities $\left|E / E_{0}\right|^{2}$ for scaling factor $S=1$, where $\left|E_{0}\right|$ denotes the incident field amplitude. (E) The envelope of metapixel reflectance amplitudes reproduces the absorption fingerprint of an adjacent model protein layer (top inset). (F) Conceptual sketch of a molecule-specific barcode produced by imaging-based readout of the metasurface's reflectance response. Image regions 1 and 2 indicate the spatially-encoded vibrational information from the corresponding metapixel resonances in panel $(\mathrm{E})$. 
A pixelated dielectric metasurface design consisting of an array of $10 \times 10$ metapixels was fabricated using electron-beam lithography and reactive ion beam etching. Ellipse axes and unit cell periodicities are identical to the values given for the numerical simulations in Fig. 1. The scaling of the resonator unit cell was linearly interpolated between factors of $S=1.00$ and $\mathrm{S}=1.34$ in 100 steps. A fixed metapixel size of $100 \mu \mathrm{m}$ x $100 \mu \mathrm{m}$ yields a total metasurface area of around $2.25 \mathrm{~mm}^{2}$ (Fig. 2A). Sizes are chosen to provide a trade-off between metapixel signalto-noise ratio and number of pixels (Fig. S2). Analysis of scanning electron microscopy (SEM) images captured for multiple metapixels confirms the accurate reproduction of the resonator design as well as the linear scaling of the unit cell geometry over the entire metasurface area (Fig. 2B, Fig. S3).

Optical characterization of the metasurface is performed in reflection using a QCL-based mid-IR microscopy system equipped with a 480 x 480 pixel array-based imaging detector. We utilize a refractive $4 \mathrm{X}$ objective with a large $2 \mathrm{~mm}$ field of view (FOV), allowing us to acquire the optical response of all metasurface pixels simultaneously (Fig. 2C). Reflectance images captured for different wavenumbers of the incident mid-IR radiation are shown in Fig. 2D. At each incident wavenumber, high reflectance intensity indicates the excitation of a metapixel with matching resonance frequency in a specific spatial location on the metasurface, experimentally confirming the one-to-one mapping between metapixel location and frequency of resonant vibrational enhancement.

The spectra of 21 metapixels and extracted resonance positions of all 100 metasurface pixels are shown in Fig. 2E and Fig. 2F. The fabricated pixelated metasurface delivers resonances with average FWHM of $13.7 \mathrm{~cm}^{-1}$ and continuous tuning of the resonance frequency over the full amide band range from $1370 \mathrm{~cm}^{-1}$ to $1770 \mathrm{~cm}^{-1}$, corresponding to a spectral resolution of $4 \mathrm{~cm}^{-1}$. Each individual metapixel provides an experimentally measured resonance Q-factor above 100 with an average of $\mathrm{Q}=115$ (Fig. S4), which constitutes an improvement of more than one order of magnitude over antenna geometries based on gold nanostructures $(18,19)$. Importantly, our nanophotonic design can easily be extended to cover the entire mid-IR molecular absorption band region by increasing the range of geometrical scaling parameters (Fig. S5). 

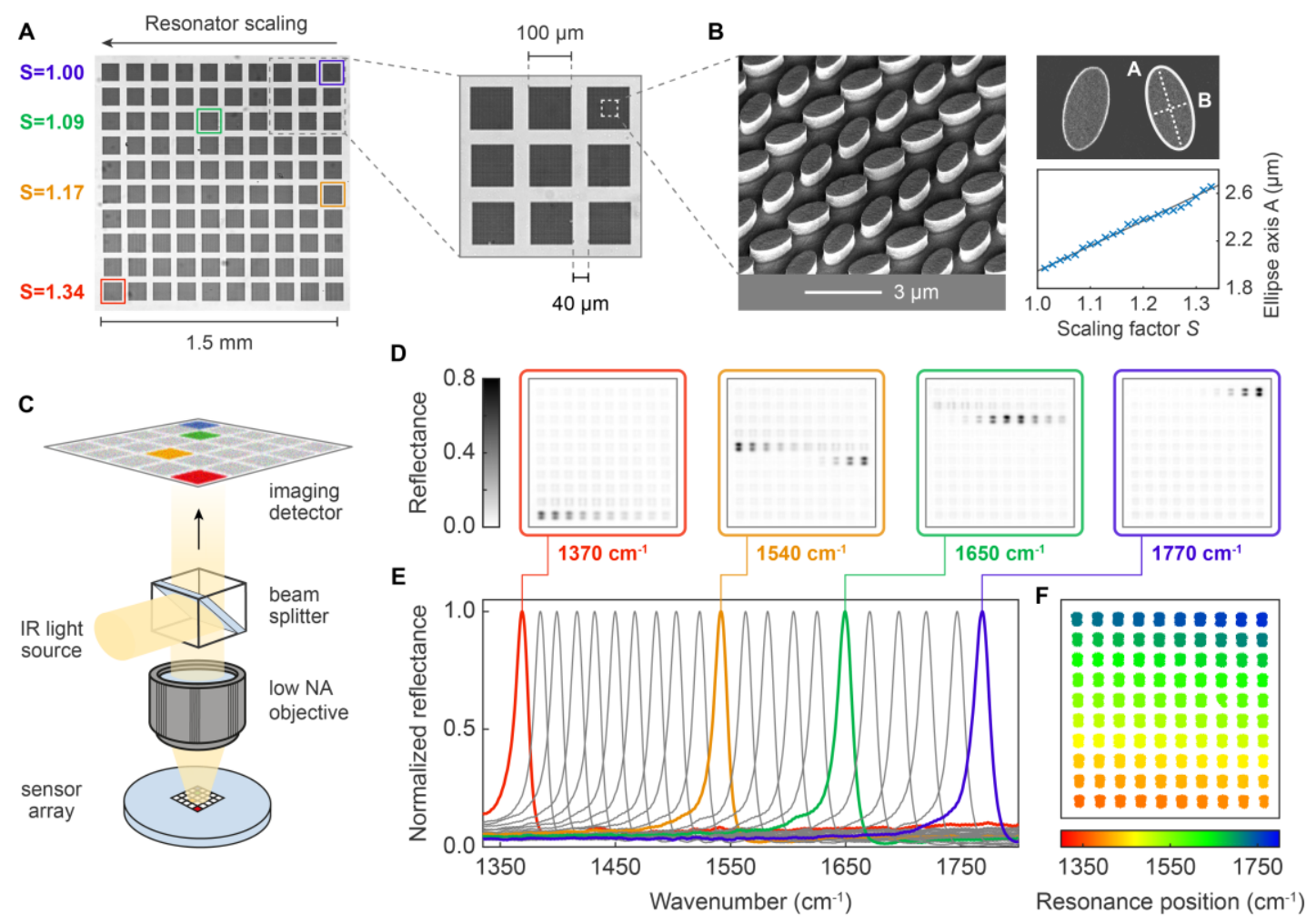

Fig. 2: Experimental realization of the pixelated metasurfaces. (A) Optical micrographs of the fabricated 100 pixel metasurface. (B) SEM micrographs confirm the linear relationship between scaling factor and ellipse feature size. (C) Sketch of the imaging-based mid-IR microscopy system. (D) Reflectance images of the pixelated metasurface recorded at four specific wavenumbers in the mid-IR spectral range. (E) Normalized reflectance spectra for 21 of the 100 metapixels. Resonance positions of the colored curves correspond to the respective reflectance images in panel $(\mathrm{E})$. (G) Extracted resonance positions for all metapixels demonstrate continuous spectral coverage from $1370 \mathrm{~cm}^{-1}$ to $1770 \mathrm{~cm}^{-1}$.

We experimentally demonstrate the amplification and detection of molecular fingerprints by interrogating a physisorbed monolayer of recombinant protein $A / G$, focusing specifically on its amide I and II absorption signature. Metapixel reflectance spectra before and after the protein $\mathrm{A} / \mathrm{G}$ physisorption are presented in Figs. 3A and 3B, respectively. All spectra are normalized to the peak reflectance values of the reference measurement without analyte.

Addition of the protein $\mathrm{A} / \mathrm{G}$ monolayer strongly modulates the metapixel reflectance spectra, with a pronounced maximum reflectance intensity decrease of $25 \%$. The absorbance signal calculated from the peak reflectance envelopes before $\left(\mathrm{R}_{0}\right)$ and after physisorption $\left(\mathrm{R}_{\mathrm{S}}\right)$ clearly reveals the characteristic amide I and II absorption signature of the protein A/G molecules (Fig. 3C). Furthermore, the high absorbance signal of up to A=140 mOD extracted from a protein monolayer demonstrates the strong vibrational enhancement of our metasurface design, which exceeds the performance of state-of-the-art periodicity optimized metal antenna geometries (20) by more than one order of magnitude (Fig. S7). Combined with an experimental noise level of $1.8 \mathrm{mOD}$, this value corresponds to a detection limit of 2130 molecules per $\mu \mathrm{m}^{2}$ (Fig. S6). 


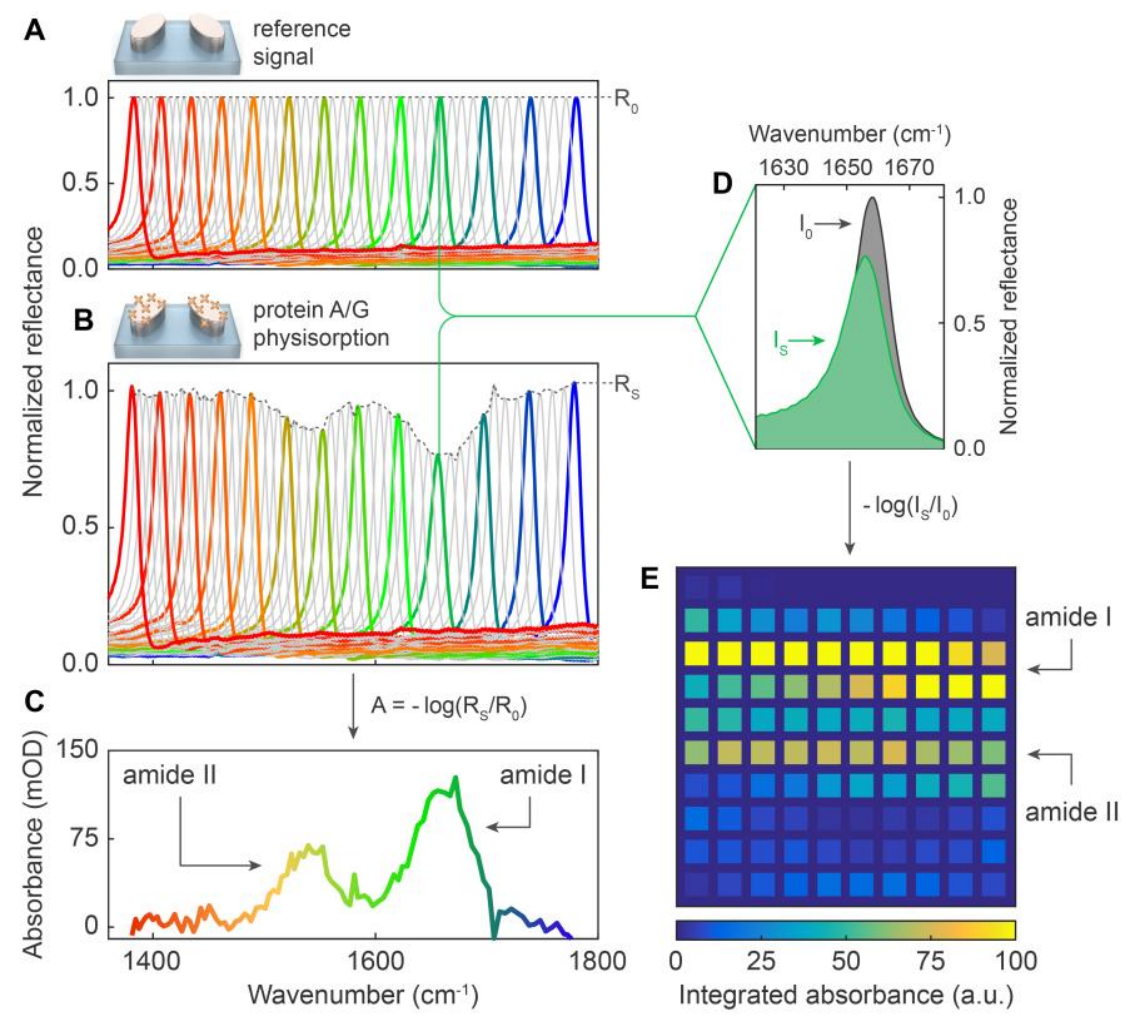

Fig. 3: Molecular fingerprint retrieval and spatial absorption mapping. (A) Normalized metapixel reflectance spectra for a reference measurement prior to protein physisorption. The dashed vertical line indicates the envelope of peak reflectance amplitudes $\mathrm{R}_{0}$ resulting from all metapixels. (B) Normalized metapixel reflectance spectra after physisorption of a protein $A / G$ monolayer including peak reflectance envelope $R_{S}$ (dashed line). (C) Protein absorption fingerprint calculated from the reflectance envelopes $R_{0}$ and $R_{S}$. (D) Broadband operation of the metasurface can be emulated by considering the integrated reflectance signal for all pixels. (E) Spectral integration translates the absorption signature from panel (C) into a spatial absorption map, which features the amide I and II bands as distinct high intensity regions of the resulting molecular barcode.

Progress in sensing technology is driven by a need for miniaturization, with the ultimate goal of realizing a mid-IR "sensor-on-a-chip" platform, which combines high sensitivity, portability, robustness, and ease of use (21). Such miniaturization is a particular challenge in the mid-IR, due to the need for Fourier-transform IR (FTIR) spectrometers or frequency scanning laser sources $(22,23)$. To address this, approaches based on microelectromechanical systems (MEMS) and optical waveguides have been explored (24-26), but still require moving parts or complex coupling optics.

These limitations can be overcome by combining our pixelated sensor metasurface with a broadband light source and a broadband IR imaging detector such as a high-resolution microbolometer or a mercury cadmium telluride (MCT) focal-plane array.. Here, we consider one possible implementation of this general concept, where the signal from each sensor 
metapixel is imaged on a corresponding detector pixel of the imaging chip. Importantly, our sensor metasurface can also be operated in transmission, enabling full chip integration via the direct stacking of sensor metasurface and detector chip (Fig. S9).

To assess the capability of our metasurface for imaging-based spectrometer-less fingerprint detection, we calculate the integrated reflectance signal from the spectral data of each metapixel. These integrated signals are analogous to a readout of the sensor metasurface's optical response with a broadband detector chip before $\left(\mathrm{I}_{0}\right)$ and after $\left(\mathrm{I}_{\mathrm{S}}\right)$ addition of the protein layer (Fig. 3D), which can be utilized to calculate the absorbance for all metapixels via $A=-\log \left(I_{S} / I_{0}\right)$.

The resulting spatial absorption map associated with the physisorption of a protein $A / G$ monolayer clearly shows the spectral location and relative intensity distribution of the characteristic amide I and II absorption bands as two distinct high signal regions of the image (Fig. 3E). This image, representing a molecular barcode, carries the spectral fingerprint information of the adsorbed molecules, providing chemically specific detection in a chipintegrated design and without the need for spectrometry. This functionality is crucially enabled by the spectrally clean high-Q resonances of the dielectric metapixels and cannot be achieved with metapixels based on metal antennas due to linewidth limitations (Fig. S8).

To demonstrate the versatility of our approach, we measure and compare the molecular barcodes of protein $\mathrm{A} / \mathrm{G}$, a mixture of the polymers polymethyl methacrylate (PMMA) and polyethylene (PE), and glyphosate herbicide, covering applications in fields as diverse as biosensing, materials science and environmental monitoring. In all three cases, the barcode matrices feature mutually distinct high intensity image regions unique to the vibrational signature of the investigated molecules (Fig. 4A), underscoring the chemical identification capability of our method. 


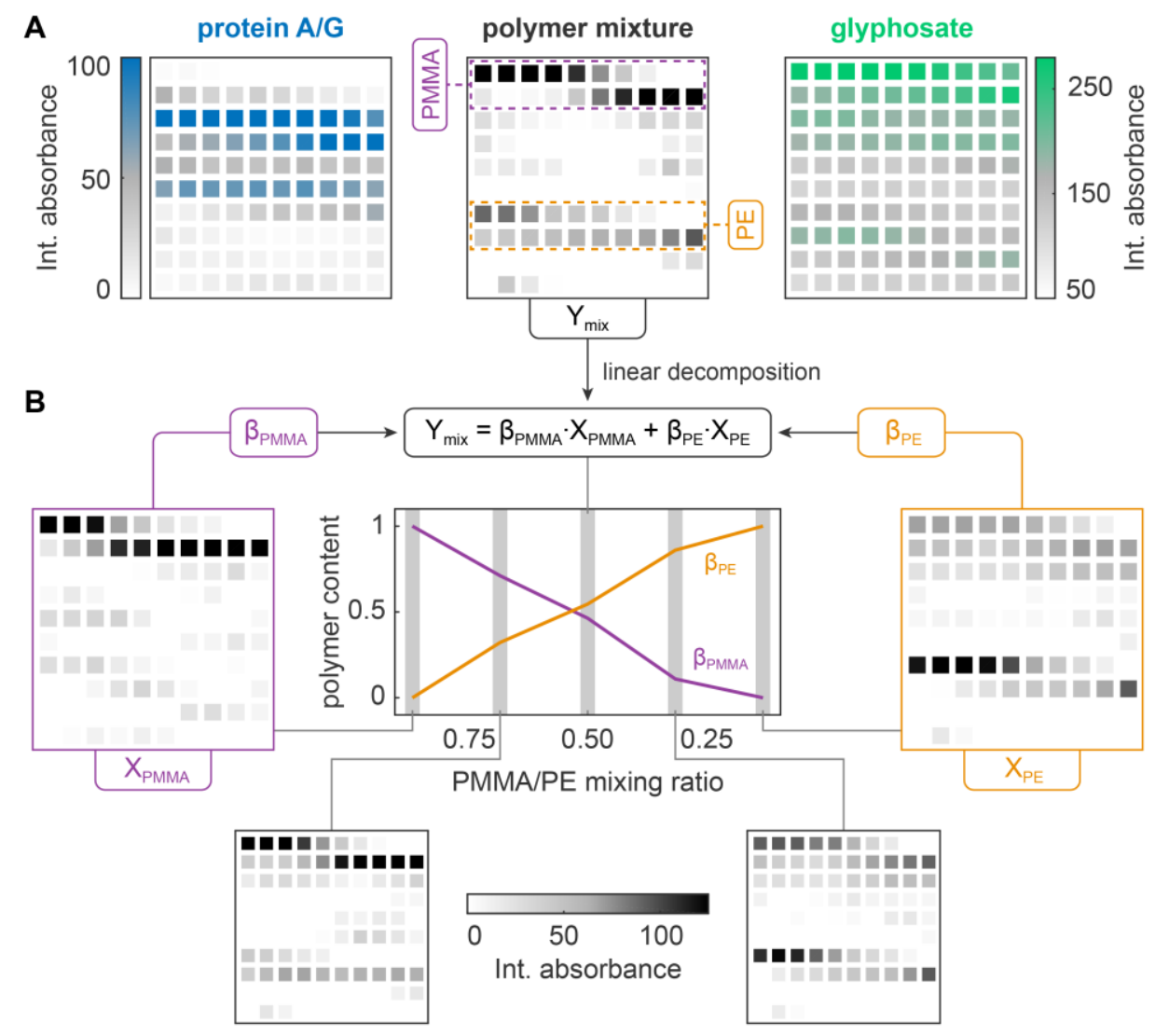

Fig. 4: Imaging-based chemical identification and compositional analysis. (A) Molecular barcodes of protein A/G, a mixture of PMMA and PE polymers, and glyphosate herbicide clearly reveal the distinct absorption fingerprints of the analytes. (B) Barcode matrices for PMMA/PE polymer mixtures with several mixing ratios. Linear decomposition analysis of all mixing states $Y$ with respect to the pure PMMA and PE barcode matrices confirms accurate readout of the deposited polymer ratios.

Since the spatial absorption map acts as a barcode of the molecular fingerprint, it offers the potential for the chemical identification of arbitrary analyte compositions through pattern recognition based on a library of such molecular barcode signatures. To elucidate the power and simplicity of this approach, we apply the sensor metasurface to detect a series of predefined mixtures of PMMA and PE polymer molecules deposited on the metasurface. Figure 4B shows molecular barcodes for pure PMMA and PE as well as PMMA/PE mixing ratios of 0.25, 0.50, and 0.75 . The characteristic molecular signatures of PMMA and PE appear as distinct image features in the top and bottom halves of the barcode matrix, respectively. When increasing the relative amount of PE in the mixture, we observe a substantial increase of the PE signal versus mixing ratio combined with an associated decrease of the PMMA signal, confirming the compositional sensitivity of our method.

We carry out further image-based analysis by decomposing the barcode matrices of individual mixing states into a linear combination of the pure PMMA and PE molecular barcodes (Fig. 4B, center). Specifically, we solve the equation 


$$
Y=\beta_{\mathrm{PMMA}} \cdot X_{\mathrm{PMMA}}+\beta_{\mathrm{PE}} \cdot X_{\mathrm{PE}}
$$

for each of the input mixing states $Y$, where $X_{\mathrm{PMMA}}, X_{\mathrm{PE}}$ are the input barcodes of the pure materials and $\beta_{\mathrm{PMMA}}, \beta_{\mathrm{PE}}$ are the output coefficients associated with the analyte content on the surface. We find that the PMMA and PE polymer amounts obtained from our image decomposition analysis accurately capture the linear variation of the polymer composition, highlighting the rich chemical and compositional information available from such absorption maps.

The presented nanophotonic technique redefines the prospects of infrared absorption spectroscopy by overcoming resonance-linewidth limitations and the need for complex instrumentation. Our high-Q pixelated dielectric metasurface design is capable of translating molecular fingerprint information into an image-based molecular barcode, enabling chemically specific and compositionally sensitive detection. Sensitivity and Q-factor of our metasurface concept can be further improved by decreasing the resonator orientation angle, only limited by the inhomogeneity of the underlying nanofabrication; even stronger near-field enhancement could be achieved by using more sophisticated designs other than ellipses for meta-atoms. Crucially, our Si-based pixelated metasurface design is compatible with industry standard CMOS technology, allowing for the low-cost wafer-scale fabrication of sensor chips for practical applications. Going beyond simple linear decomposition techniques, our molecular barcoding technique offers unique possibilities for further analysis using neural network-based image recognition methods and machine learning $(27,28)$ especially when considering non-linear analyte association processes, paving the way towards versatile and highly sensitive chipintegrated mid-infrared spectroscopy devices. 


\section{References and Notes}

1. B. H. Stuart, Infrared Spectroscopy: Fundamentals and Applications (John Wiley \& Sons, Ltd, 2005).

2. D. Dregely, F. Neubrech, H. Duan, R. Vogelgesang, H. Giessen, Nat. Commun. 4, 2237 (2013).

3. O. Limaj et al., Nano Lett. 16, 1502-1508 (2016).

4. C. Huck et al., ACS Nano 8, 4908-4914 (2014).

5. F. Neubrech, C. Huck, K. Weber, A. Pucci, H. Giessen, Chem. Rev. 117, 5110-5145 (2017).

6. L. Dong et al., Nano Lett. 17, 5768-5774 (2017).

7. B. Cerjan, X. Yang, P. Nordlander, N. J. Halas, ACS Photonics 3, 354-360 (2016).

8. A. I. Kuznetsov, A. E. Miroshnichenko, M. L. Brongersma, Y. S. Kivshar, B. Luk'yanchuk, Science 354, aag2472 (2016).

9. A. Y. Zhu, A. I. Kuznetsov, B. Luk'yanchuk, N. Engheta, P. Genevet, Nanophotonics 6, 1-20 (2017).

10. A. Arbabi, Y. Horie, M. Bagheri, A. Faraon, Nat. Nanotechnol. 10, $937-943$ (2015).

11. M. Decker et al., Adv. Opt. Mater. 3, 813-820 (2015).

12. M. Khorasaninejad et al., Science 352, 1190-1194 (2016).

13. D. Lin, P. Fan, E. Hasman, M. L. Brongersma, Science 345, 298-302 (2014).

14. M. Caldarola et al., Nat. Commun. 6, 7915 (2015).

15. M. F. Limonov, M. V. Rybin, A. N. Poddubny, Y. S. Kivshar, Nat. Photonics 11, 543-554 (2017).

16. C. Wu et al., Nat. Commun. 5, 3892 (2014).

17. S. Campione et al., ACS Photonics 3, 2362-2367 (2016).

18. Kai Chen, R. Adato, H. Altug, ACS Nano, 7998-8006 (2012).

19. C. Wu et al., Nat. Mater. 11, 69-75 (2011).

20. S. Bagheri et al., ACS Photonics 2, 779-786 (2015).

21. H. Lin et al., in Proc. SPIE 10249, Integrated Photonics: Materials, Devices, and Applications IV (2017), p. 102490G.

22. M. J. Baker et al., Nat. Protoc. 9, 1771-1791 (2014).

23. F. K. Tittel, D. Richter, A. Fried, in Solid-State Mid-Infrared Laser Sources, I. T.

Sorokina, K. L. Vodopyanov, Eds. (Springer Berlin Heidelberg, Berlin, Heidelberg, 2003), pp. $458-529$.

24. D. Khalil et al., in Proc. SPIE 7930, MOEMS and Miniaturized Systems X (2011), p. 79300J. 
25. A. L. Holsteen, S. Raza, P. Fan, P. G. Kik, M. L. Brongersma, Science 358, 1407-1410 (2017).

26. D. M. Kita et al., IEEE J. Sel. Top. Quantum Electron. 23, 340-349 (2017).

27. G. E. Hinton, Science 313, 504-507 (2006).

28. Y. LeCun, Y. Bengio, G. Hinton, Nature 521, 436-444 (2015).

29. D. Rodrigo et al., Science 349, 165-168 (2015).

30. X. Gai, D.-Y. Choi, B. Luther-Davies, Opt. Express 22, 9948 (2014).

31. P. Bassan, M. J. Weida, J. Rowlette, P. Gardner, Analyst 139, 3856-3859 (2014).

32. H. P. Erickson, Biol. Proced. Online 11, 32-51 (2009).

Acknowledgments: The authors would like to thank Rui Guo and Eduardo Romero Arvelo for useful discussions. We acknowledge École Polytechnique Fédérale de Lausanne and Center of MicroNano Technology for nanofabrication. Sample fabrication was performed in part at the ACT node of the Australian National Fabrication Facility. Funding: The research leading to these results has received funding from European Research Council (ERC) under grant agreement no. 682167 VIBRANT-BIO and the European Union Horizon 2020 Framework Programme for Research and Innovation under grant agreement no. 665667 (call 2015). The authors acknowledge the support of the Australian Research Council (ARC). Author contributions: (TODO) Competing interests: The authors declare no competing interests. Data and materials availability: The authors declare that the data supporting the conclusions of this study are available within the article and its supplement. Additional data are available from the corresponding author upon reasonable request. 


\section{Supplementary Materials:}

Materials and Methods

Supplementary text

Figures S1-S9

References (30-32) 


\section{Materials and methods}

Numerical calculations: Simulations of the metasurface optical response were performed using the frequency domain finite element (FEM) Maxwell solver contained in CST STUDIO SUITE 2017 and the unit cell geometry was approximated using a tetrahedral mesh. Base values of the resonator geometrical parameters were defined as $\mathrm{Px}=3.92 \mu \mathrm{m}, \mathrm{Py}=2.26 \mu \mathrm{m}$ for the unit cell periodicities, and $\mathrm{A}=1.96 \mu \mathrm{m}, \mathrm{B}=0.96 \mu \mathrm{m}$ for the ellipse long and short axes, with an orientation angle of $\theta=20^{\circ}$. The height of the resonators was fixed at $\mathrm{H}=700 \mathrm{~nm}$. To vary the resonance frequency of the resonators, all unit cell dimensions except resonator height were scaled with a factor $\mathrm{S}$, interpolated between 1.00 and 1.30 in 25 steps. Refractive index values for the hydrogenated amorphous silicon $(\mathrm{a}-\mathrm{Si}: \mathrm{H})$ resonators were taken from mid-infrared ellipsometry measurements carried out on a $700 \mathrm{~nm}$ thick a-Si:H film on a $\mathrm{MgF}_{2}$ substrate, yielding average values of $\mathrm{n}=3.21$ and $\mathrm{k}=0.00$ in the target spectral range. The refractive index of $\mathrm{MgF}_{2}$ was taken as $\mathrm{n}=1.31$. To demonstrate protein detection, the chip surface was covered with a $2.5 \mathrm{~nm}$ thick conformal model protein layer. The refractive index of this layer was described using a 2-Lorentzian protein permittivity model with parameter values taken from (29).

Pixelated metasurface fabrication: All sample fabrication was carried out on magnesium fluoride $\left(\mathrm{MgF}_{2}\right)$ chips, which were chosen due to their low absorption and low refractive index in the mid-IR spectral range. A hydrogenated amorphous silicon (a-Si:H) layer of $700 \mathrm{~nm}$ thickness was deposited onto the chips by plasma-enhanced chemical vapour deposition as described previously (30), followed by the spin coating of a double layer of polymethyl methacrylate (PMMA) resist of different molecular weights (495K and 950K). The elliptical resonator pattern

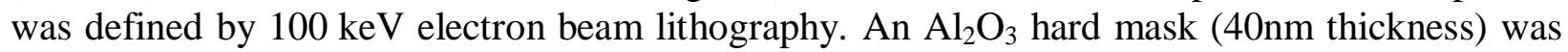
produced via evaporation and wet-chemical lift-off and the ellipse pattern was subsequently transferred into the underlying a-Si:H layer by fluorine-based dry plasma etching. Finally, the $\mathrm{Al}_{2} \mathrm{O}_{3}$ hard mask was removed by 2 min of RCA 1 wet etching (water, ammonium hydroxide and hydrogen peroxide in ratio $5: 1: 1)$ at $80^{\circ} \mathrm{C}$.

Imaging-based metasurface measurements: The optical response of our metasurface chips was characterized with a Spero laser-based spectral imaging microscope (Daylight Solutions Inc., San Diego, CA, USA). The microscope is equipped with four quantum cascade laser heads, which allow continuous spectral tuning from $946 \mathrm{~cm}^{-1}$ to $1800 \mathrm{~cm}^{-1}$. For imaging, a low magnification objective (4X, $0.15 \mathrm{NA}$ ) was used, which covers a large $2 \times 2 \mathrm{~mm}^{2}$ field of view (FOV) and delivers $24 \mu \mathrm{m}$ diffraction limited spatial resolution at $1655 \mathrm{~cm}^{-1}$. For a full description of the Spero microscopy system see Ref. (31). All optical measurements of the metapixel array were carried out in reflection mode and normalized to the reflection signal of a plain gold mirror. Measurements were performed in the spectral range from $1300 \mathrm{~cm}^{-1}$ to $1800 \mathrm{~cm}^{-1}$ with $0.5 \mathrm{~cm}^{-1}$ spectral resolution. To address backscattering effects from the $\mathrm{MgF}_{2}$ substrate, a background measurement is taken on an empty area of the chip, spatially filtered to remove surface impurities, and subtracted from the metapixel array data. A low pass filter is applied to decrease interference effects from the laser system. During each measurement, $480 \mathrm{x}$ 480 pixel reflectance images are captured for each laser frequency point in the target spectral range. To obtain reflectance spectra for individual metapixels, we first locate the image pixels 
corresponding to the area of an individual metapixel. Subsequently, the spectrally resolved reflectance data from these image pixels is averaged to yield the full metapixel spectrum. To obtain the integrated absorbance signals displayed in the molecular barcode matrices, we performed trapezoidal numerical integration of the reflectance spectra over the range from $1300 \mathrm{~cm}^{-1}$ to $1800 \mathrm{~cm}^{-1}$. The influence of side band reflection signals was reduced by applying a Gaussian band pass filter centered on the resonance position. On average, dust or other analyte impurities produced absorbance signal outliers for 1 of 100 pixels per measurement. Affected pixels were excluded from the absorption maps. Due to the high number of metasurface pixels, a moving mean filter with a filter width of 4 could be applied to improve the clarity of the absorption maps.

Analyte preparation. For the chemical identification measurements, protein $\mathrm{A} / \mathrm{G}$ was diluted in $10 \mathrm{mM}$ acetate solution at $0.5 \mathrm{mg} / \mathrm{mL}$ concentration. The sensor chip was incubated with protein A/G solution to allow protein physisorption, followed by rinsing with deionized water to remove unbound protein and agglomerates. Glyphosate pesticide was diluted in deionized water at $5 \mathrm{mg} / \mathrm{ml}$ concentration and spin coated on the sample at $6000 \mathrm{rpm}$ spin speed. Polymethyl methacrylate with average molecular weight of 350,000 and medium density polyethylene were deposited by thermal evaporation. The deposition rate and layer thickness was measured with quartz crystal oscillator. Layer thicknesses for pure PMMA and PE before mixing were $10 \mathrm{~nm}$ and $40 \mathrm{~nm}$, respectively. 


\section{Supplementary text}

\section{Theoretical analysis of the high- $Q$ metapixel design based on zig-zag arrays}

The high-Q metasurface design utilized in this work is based on zig-zag arrays of elliptical dielectric meta-atoms, where the collective resonance is formed by the electric dipole modes polarized along the long axis of each individual meta-atom. For a lossless system, the linewidth of the resonance depends on the scattering loss, which is determined by the overlapping of the mode profile and the field polarization of the scattering channels. For the periodic zig-zag metasurface studied here, the scattering channels are the zeroth order plane waves propagating in the normal direction; the overlapping of the mode profile and the plane wave is determined by the net-electric dipole moment in each unit cell.

The zig-zag design allows us to precisely control this overlap by fine-tuning the unit cell geometry including the orientation angle $\theta$ of the meta-atoms. When the collective mode is excited, the dominant component of the dipole moments of the meta-atoms $\left(p_{\mathrm{y}}\right)$ are actually perpendicular to the incident field polarization $E_{x}^{i}$ as shown in Fig. S1A. However, due to the antisymmetric distribution of the components $\left(p_{1, y}=-p_{2, y}\right)$, the out-coupling with the plane wave of $E_{y}$ polarization is forbidden, and thus the overall scattering loss of the collective mode is suppressed significantly. In fact, only the $p_{\mathrm{x}}$ component is nonzero $\left(p_{1, \mathrm{x}}=p_{2, \mathrm{x}}\right)$ and therefore contributes to the scattering loss.

For simplicity, we assume that the zig-zag array is lossless and positioned in a homogenous background. The forward and backward scattered fields are contributed by the collective dipole resonance with nonzero net dipole moment $p_{x}$, which can be expressed as

$$
\begin{aligned}
E^{f} & =E_{x}^{i}+\frac{i \omega \eta}{2 A} p_{x}, \\
E^{b} & =\frac{i \omega \eta}{2 A} p_{x},
\end{aligned}
$$

where $\eta$ is the wave impedance of the surroundings and $A$ is the area of the unit cell. The net dipole moment within a unit cell can be further expressed as

$$
p_{x}=i 2 I u \sin \theta / \omega,
$$

where $u$ is the normalized dipole moment of a single meta-atom. $I$ is the corresponding frequency-dependent current amplitude that captures the resonant behavior, which can be calculated via

$$
I=\frac{E_{x}^{i} u \sin \theta}{Z},
$$

with $Z$ being the effective impedance of the meta-atom under the collective resonance, taking into account all the mutual interaction. Generally, the effective impedance around a single resonance can be approximated with an RLC circuit:

$$
Z=-i \omega L-\frac{1}{i \omega C}+R,
$$

where $R$ is the effective resistance and is contributed solely by the radiative loss in a lossless system. From energy conservation, it requires that 


$$
\left|E_{x}^{i}\right|^{2}=\left|E^{f}\right|^{2}+\left|E^{b}\right|^{2} .
$$

Substituting Eqs. (1a) to (4) into Eq. (5), and using the identity: $\operatorname{Re}(1 / Z)=\operatorname{Re}\left(Z^{*}\right) /|Z|^{2}=$ $R /|Z|^{2}$, we obtain the following relation between the effective resistance $R$ and the orientation angle $\theta$ :

$$
R=\frac{2 \eta u^{2} \sin ^{2} \theta}{A} .
$$

Since the quality factor $Q=R^{-1} \sqrt{L / C}$ and the effective inductance $L$ and capacitance $C$ do not change significantly when $\theta$ is small, it is expected that the quality factor $Q \propto 1 / \sin ^{2} \theta$, which grows dramatically as the orientation angle $\theta$ decreases.

To confirm the effect, we simulate the resonance behavior using the geometrical parameters from Fig. 1 of the main text with a scale factor $\mathrm{S}=1.0$; by changing the orientation angle while keeping other parameters unchanged, we clearly observe the asymptotic behavior of the quality factor as $\theta$ decreases (Fig. S1B). Although in practice, the Q-factor achievable is limited by various factors including material loss and finite sample size, our zig-zag concept provides a straightforward way to control the Q-factor and can be easily applied to meta-atom designs other than ellipses.

In general, the higher Q-factors associated with lower orientation angles increase the sensitivity of the resonators to changes in the environment, leading to improved fingerprint detection performance. On the other hand, this increased sensitivity also makes the system more susceptible to small variations in structure size associated with nanofabrication inhomogeneity. In experiments, an orientation angle of $\theta=20^{\circ}$ was chosen to provide a trade-off between these two factors by delivering experimental Q-factors above 100 within our fabrication tolerances.

Unlike many geometries based on leaky guiding modes where the electric field is mostly confined within the high-index material, this particle-based collective dipole resonance allows strong near-field enhancement at the surface of the meta-atom, which is a desirable feature for surface-based molecular sensing. Figure S1C shows the enhancement of the electric near-field intensity on two different cut-planes: one is a horizontal cut-plane $2.5 \mathrm{~nm}$ away from the substrate, the other is a vertical cut-plane across the long-axis of the meta-atom. We find strongly enhanced electric near-fields in between the meta-atoms, with electric near-field intensity enhancement values of up to 1500 .

Figure S1D again highlights the near-field intensity on the meta-atom surface, where we plot the amplitude distribution along the three line-cuts shown in Figure S1C. Note that $\mathrm{z}=0 \mathrm{~nm}$ and $\mathrm{z}=700 \mathrm{~nm}$ represent the surface of the substrate and the top surface of the meta-atoms, respectively; we choose $\mathrm{z}=2.5 \mathrm{~nm}$ and $\mathrm{z}=702.5 \mathrm{~nm}$ to match the thickness of the molecule layer.

Significantly, the enhanced near-fields are localized in distinct hot-spots on the surface of the meta-atoms, which is a characteristic feature of nanophotonic systems based on localized resonators. During sensing experiments, the target analytes interact with both the low and high intensity regions of the electric field around the meta-atom. Therefore, the effective enhancement of the molecular absorption signature is determined by the averaged electric near-field intensities around the meta-atom. We estimate this effect by averaging the simulated electric near-field intensities over a $2.5 \mathrm{~nm}$ thick layer around the meta-atoms and obtain an effective enhancement factor of 320 for a scaling factor of $S=1.0$. The reduced effective enhancement compared to the 
hot-spot values is common to all localized resonance geometries and is similarly found in plasmonic platforms (5).

These performance considerations are valid as long as the analyte presented to the surface exhibits sufficient spatial uniformity, which is fulfilled in a multitude of analyte systems of interest such as molecular monolayers, biological species (lipids, proteins, DNA, etc.) suspended in buffer solutions, or layers of solid analytes deposited by, e.g., evaporation. Low numbers of molecules can be resolved as long as a statistical distribution over the meta-atom surface is ensured, or additional surface functionalization is employed to selectively attach the molecules in the hot-spot regions.

A

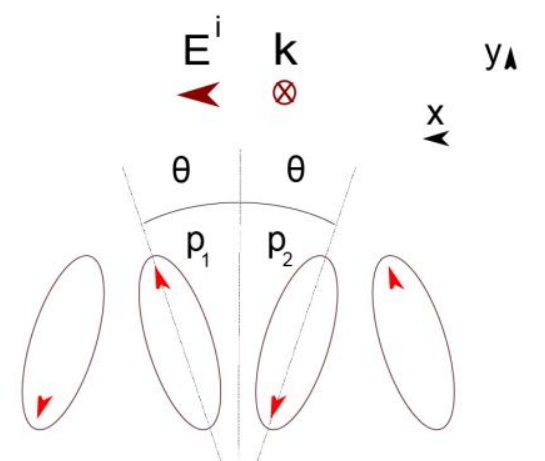

B

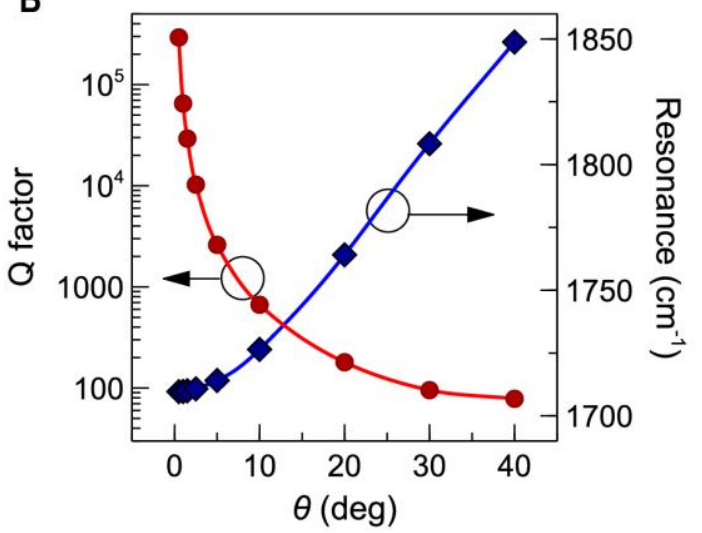

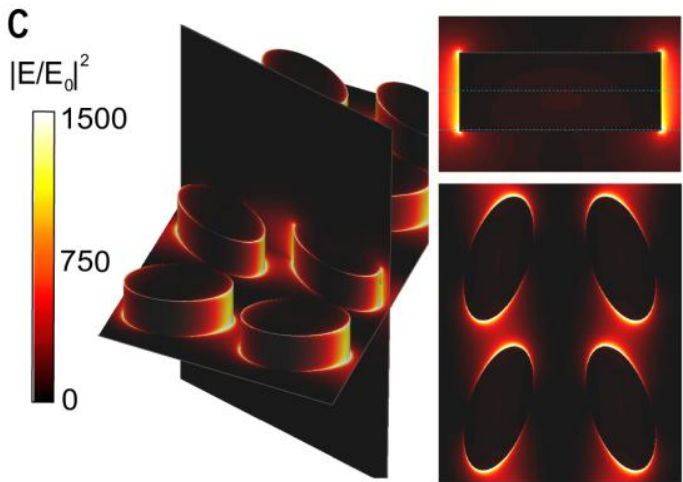

D

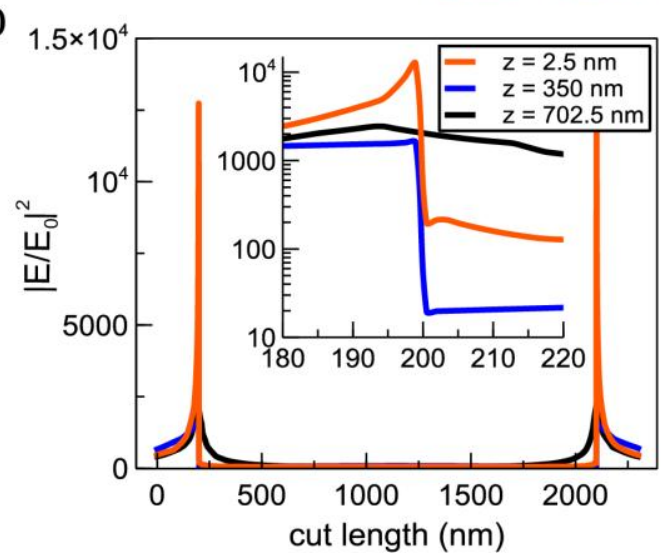

Figure S1: Theoretical analysis of high-Q collective resonance based on zig-zag arrays. (A) Schematic of the collective dipole mode of the array when excited with $E_{x}^{i}$ polarization. (B) Simulated Q-factors and resonance wavenumbers under different orientation angle $\theta$. The geometries are the same as the one simulated in Fig.1C for $S=1.0$, except for the changing orientation angle. (C) Simulated electric near-field intensity enhancement $\left|E / E_{0}\right|^{2}$ at the resonance frequency with orientation angle $\theta=20^{\circ}$, where $\left|E_{0}\right|$ denotes the incident field amplitude. (D) Field intensity $\left|E / E_{0}\right|^{2}$ enhancement along three line cuts, shown as dashed-lines in panel (C). Inset magnifies the part around the boundary between the silicon meta-atom and the surroundings. 


\section{Pixel size considerations and advanced sampling techniques}

One of the central features of our pixelated metasurface approach is the spatial encoding and separation of spectral information. Therefore, the metasurface design is crucially characterized by the number and size of individual metapixels for a given detector field of view.

Generally, a metapixel covering the detector field of view will provide the best performance in terms of signal-to-noise ratio since the analyte absorption signature is amplified and detected over the full area, but will retrieve spectral information only from a single frequency point. For higher numbers of metapixels $p$, the spectral resolution increases, but only a reduced fraction of the metasurface area (in general $1 / p$ ) will provide resonant enhancement. In experiments, metapixel sizes of $100 \mu \mathrm{m} \times 100 \mu \mathrm{m}$ were chosen to provide a trade-off between a sufficiently high signal-to-noise ratios and the number of pixels required for $4 \mathrm{~cm}^{-1}$ spectral resolution.

Due to the flexibility of our metasurface design, a variety of advanced sampling techniques can be applied to further tailor signal-to-noise ratio and spectral coverage. To illustrate two such concepts, we consider an artificial molecular fingerprint with two features (absorption bands I and II), which exhibit large spectral separation and strongly dissimilar absorption magnitudes (Fig. S2).

First, the retrieval of absorption signatures from weak molecular vibrations can be improved by utilizing metapixels with increased sizes for these frequency points, which results in higher signal-to-noise ratios as outlined above. Likewise, metapixel size can be decreased for spectral regions of strong molecular absorption, maximizing the total number of metapixels in the detector field of view.

Second, the spectral resolution of the absorption bands of interest can be increased by implementing non-uniform frequency sampling. With this approach, denser frequency sampling is employed in spectral regions with fine fingerprint features, whereas the molecular signature is sampled more broadly otherwise (Fig. S2). This technique allows to increase the spectral fidelity of the fingerprint reproduction while keeping the total number of metapixels constant.
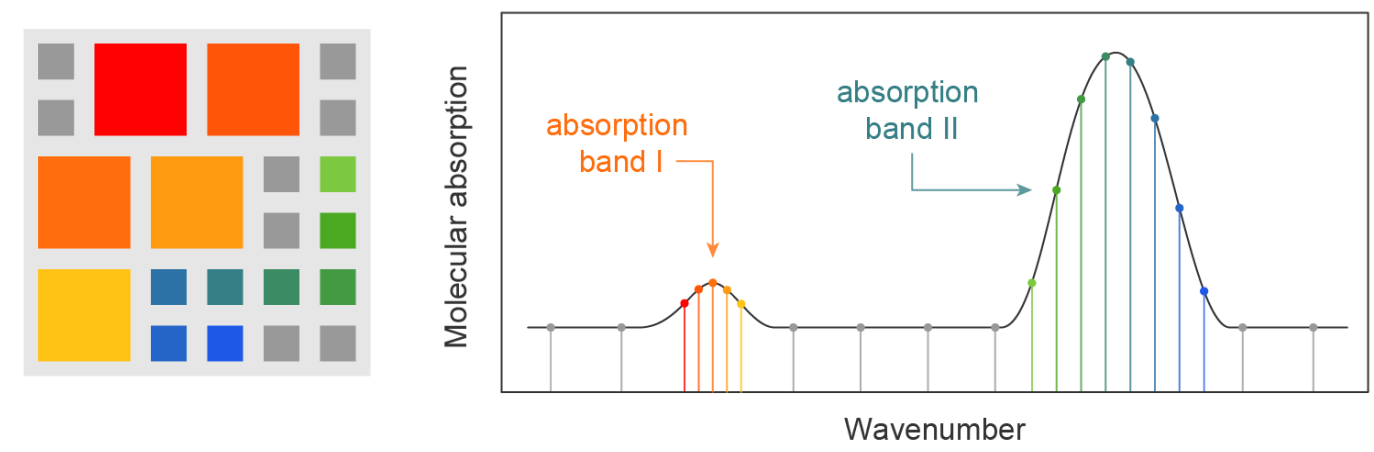

Figure S2: Advanced sampling techniques. Challenging molecular absorption signatures with large spectral separation and strongly dissimilar absorption magnitudes can be resolved by employing metapixels with tailored sizes as well as non-uniform frequency sampling. 


\section{Scanning electron microscopy characterization of the metasurface}

The fabricated pixelated metasurface was extensively characterized using scanning electron microscopy (SEM). Figure S3A shows exemplary SEM images for metapixels with the highest $(\mathrm{S}=1.36)$ and lowest $(\mathrm{S}=1.00)$ scaling factor values defined in the metasurface, confirming the exact reproduction of the target ellipse shape. To provide a thorough analysis of the resonator geometrical over the full metasurface, we extract the unit cell parameters (ellipse axes lengths and periodicities) from SEM images of 25 metapixels distributed throughout the scaling factor range (Fig. S3B). We find a nearly linear relationship between scaling factor and the geometrical parameters over the full scaling range, which confirms the accuracy and uniformity of our nanostructuring process. Furthermore, the linearity of the parameters is crucial for enabling the one-to-one mapping between spectral and spatial information demonstrated in the manuscript.

A

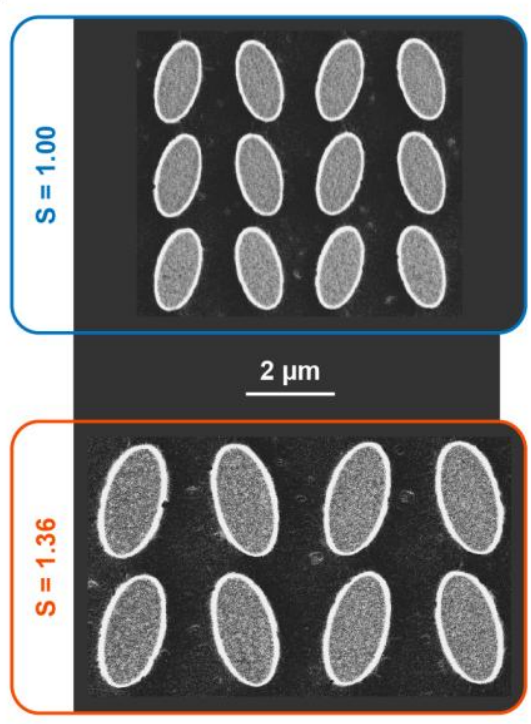

B

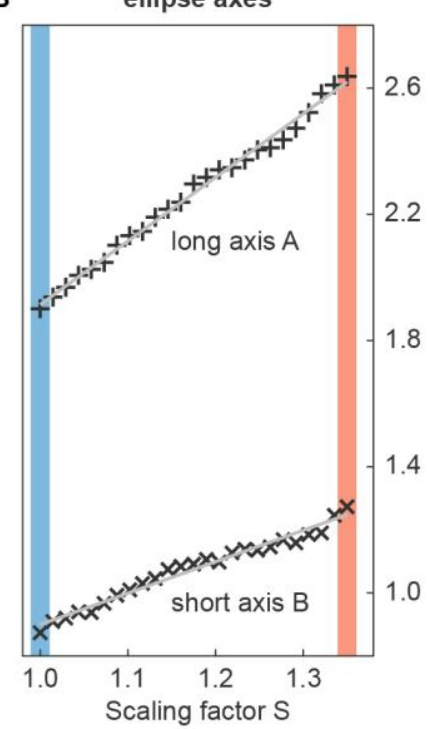

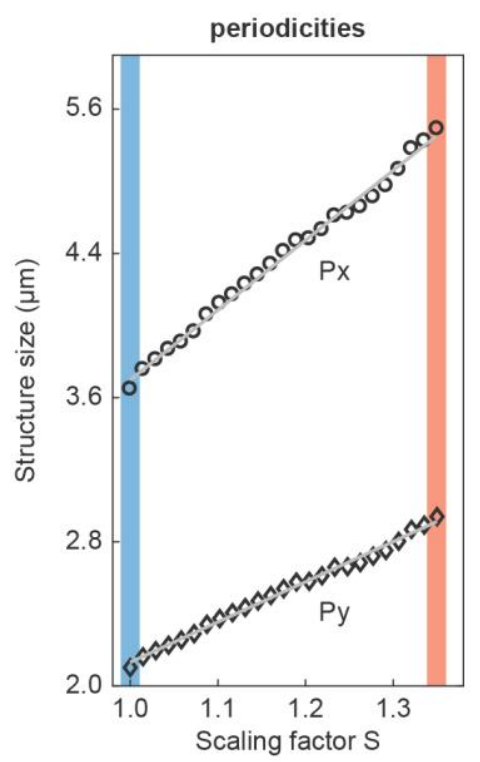

Figure S3: Scanning electron microscopy analysis. (A) Exemplary SEM images of fabricated metapixels with scaling factors of $S=1.00$ and $S=1.36$. (B) Extracted geometrical parameters of the resonators for 25 of the 100 metapixels. Grey lines represent the linear trends of the data. 


\section{Full spectral characterization of the metasurface}

Normalized reflectance spectra for all 100 pixels of the dielectric metasurface are shown in Fig. S4A. We perform full spectral characterization of the metasurface by extracting the resonance positions, linewidths, quality factors and maximum signal amplitudes from the spectral data using a standard peak fitting approach in MATLAB. Spectral properties are presented as a function of pixel number, where \#1 corresponds to a scaling factor of $S=1.0$ and $\# 100$ to $\mathrm{S}=1.34$, respectively.

We find resonance positions with continuous variation over a spectral coverage range from $1370 \mathrm{~cm}^{-1}$ to $1770 \mathrm{~cm}^{-1}$ combined with an average linewidth (full width at half maximum, FWHM) of $13.7 \mathrm{~cm}^{-1}$ (Fig. S4B,C). This results in an average Q-factor (resonance position / FWHM) of 115, highlighting the ultra-sharp nature of the dielectric resonances (Fig. 4D). Maximum peak reflectance values are shown in Fig. S4E). The decrease in reflectance for higher pixel numbers (i.e., higher scaling factors) is attributed to the relative decrease of resonator mode volume caused by the fixed resonator height $\mathrm{H}=0.7 \mu \mathrm{m}$.
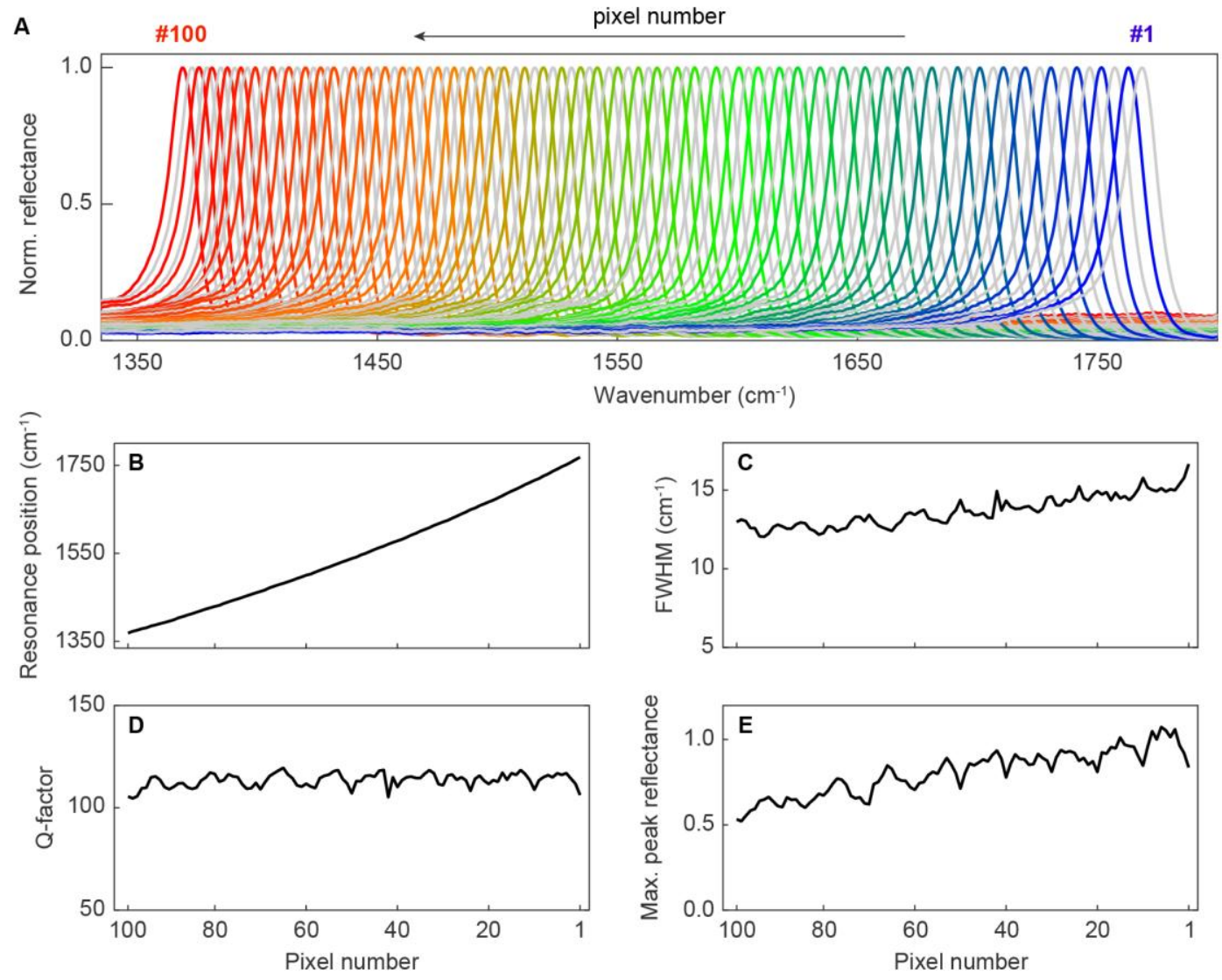

Figure S4: Spectral characterization of the pixelated dielectric metasurface. (A) Normalized reflectance spectra. (B) Resonance position. (C) Full width at half maximum (FWHM). (D) Resonance Q-factor. (E) Maximum peak reflectance. 


\section{Extension of metasurface working range}

Our versatile dielectric resonator design allows for the straightforward extension of the operating spectral range by increasing the range of scaling factors S. Figure S5A shows simulated reflectance spectra for scaling factors between $S=0.56$ and $S=2.0$, linearly interpolated in 21 steps. The geometrical parameters before scaling are identical to the design described in the manuscript with $\mathrm{Px}=3.92 \mu \mathrm{m}, \mathrm{Py}=2.26 \mu \mathrm{m}$ for the unit cell periodicities, and $\mathrm{A}=1.96 \mu \mathrm{m}$, $\mathrm{B}=0.96 \mu \mathrm{m}$ for the ellipse long and short axes, and an orientation angle of $\theta=20^{\circ}$.

We find ultra-sharp resonances covering the mid-IR molecular absorption band region from $2.5 \mu \mathrm{m}$ to $10 \mu \mathrm{m}$ together with a linear relationship between scaling factor and resonance position (Fig. S5B), which facilitates the design of suitable metapixels for arbitrary target absorption bands. The decrease in performance at a wavelength of around $5 \mu \mathrm{m}$ is due to the intrinsic absorption of a-Si in this range, and can be overcome by moving to a different resonator material such as germanium. With the exception of the a-Si absorption region, resonance sharpness $(\mathrm{Q}>100)$ is maintained over the full target wavelength range, and Q-factors above 600 can be achieved in the long wavelength region around $10 \mu \mathrm{m}$, confirming the high performance and versatility of our nanophotonic design.

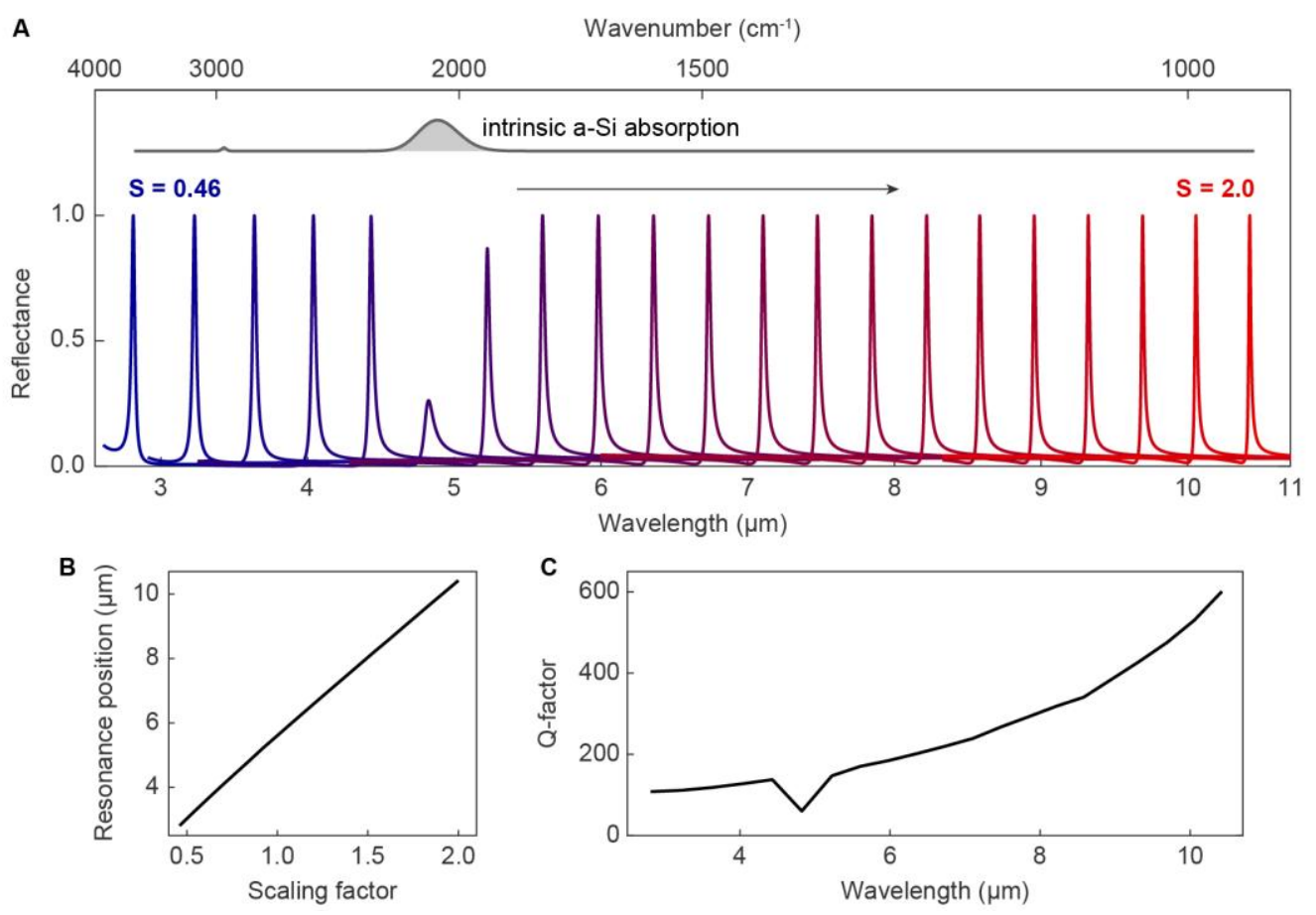

Figure S5: Extended metasurface design working range. (A) Simulated reflectance spectra for scaling factors ranging from $S=0.56$ to $S=2.0$. (B) Resonance position varies linearly with scaling factor over a wide range. (C) Q-factors remain above 100 over the entire wavelength range, with peak values above 600 in the long wavelength region around $10 \mu \mathrm{m}$. 


\section{Experimental noise and limit of detection estimate}

We estimate the limit of detection of our sensor metasurface by considering the absorbance value of $140 \mathrm{mOD}$ obtained from a protein monolayer (see Fig. 3C in the manuscript) together with the noise level of our experiments.

The experimental noise level is determined by capturing a total of $n=5$ blank metasurface measurements without the presence of any analytes. For all data sets, the envelopes of the peak reflectance values $R_{i}(i=1, \cdots, n)$ are derived as shown in the manuscript and the average value of these envelopes is calculated via $R_{\text {avg }}=\left(R_{1}+\cdots+R_{n}\right) / n$. This allows us to determine the absorbance variation $\Delta A_{i}=-\log \left(R_{i} / R_{\text {avg }}\right)$ for each measurement as shown in Fig. S6.

Each absorbance variation curve consists of $p=100$ points consistent with the 100 pixels of the metasurface design, where $\Delta A_{i}^{(k)}$ denotes the curve value at a specific pixel position with $k=1, \cdots, p$. The total noise level can be quantified by calculating and averaging the root mean square (RMS) value for all curves as shown in Eq. (7), which results in an experimental noise level value of $A_{\text {rms }}=1.8 \mathrm{mOD}$.

$$
A_{\mathrm{rms}}=\frac{1}{n} \sum_{i=1}^{n} \sqrt{\frac{1}{p} \sum_{k=1}^{p}\left(\Delta A_{i}^{(k)}\right)^{2}}
$$

In general, sensors are assumed to reliable detect signals exceeding three times the noise, which is often referred to as the $3 \sigma$ noise level. In our experiments, we observed an absorbance signal of around $A_{\text {exp }}=140 \mathrm{mOD}$ for a protein A/G monolayer, which corresponds to 55220 molecules per $\mu \mathrm{m}^{2}$ when assuming densely-packed spherical protein $\mathrm{A} / \mathrm{G}$ molecules with a diameter of $4.8 \mathrm{~nm}$ calculated from their molecular mass (32). A comparison of the signal and noise levels results in a detection limit of 2130 molecules per $\mu \mathrm{m}^{2}$ for protein $\mathrm{A} / \mathrm{G}$ detection.

Signal-to-noise ratio considerations also play an important role for the broadband operation of our metasurface-based approach, specifically when moving from illumination with a tunable but narrow-band quantum-cascade laser (QCL) to an intrinsically broadband globar source. In general, this transition is associated with a decrease of the available light intensity. However, due to the narrow-band nature of QCLs, the acquisition of the sensor metasurface response in a specific frequency range requires a large number of sequential measurements determined by the desired frequency resolution. For instance, covering the wavenumber range from $1300 \mathrm{~cm}^{-1}$ to $1800 \mathrm{~cm}^{-1}$ with $4 \mathrm{~cm}^{-1}$ resolution requires 125 sequential measurements. In broadband operation, only one single measurement is needed to capture the same information. This advantage enables an increase of the acquisition time by the same factor of 125, greatly improving the signal-tonoise ratio and compensating, in part, the decrease in the intensity of the incident light.

As detailed in Supplementary Section 2, advanced sampling techniques such as tailored pixel sizes and non-uniform sampling can be employed to further improve the signal-to-noise ratio. If additional gains are needed for a target application, high sensitivity pixelated detectors such as mercury cadmium telluride focal-plane arrays can be utilized in place microbolometer arrays. 


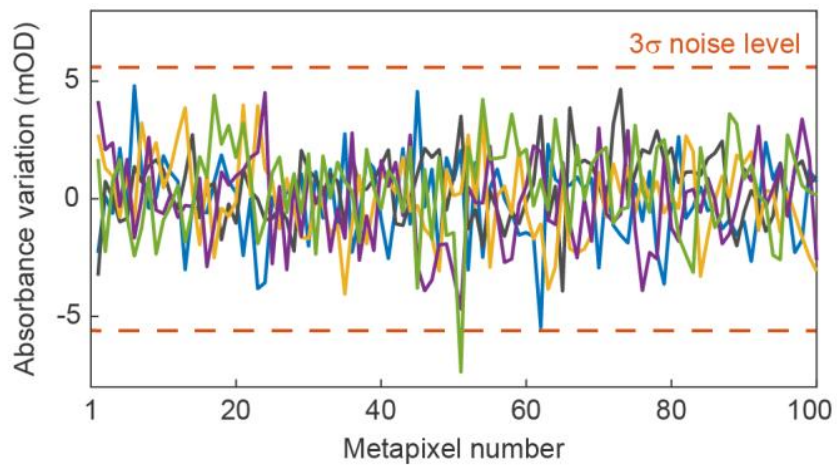

Figure S6: Experimental noise level. Absorbance variation curves derived from of $n=5$ blank metasurface measurements without the presence of any analytes. We obtain a root mean square noise value of $A_{\mathrm{rms}}=1.8 \mathrm{mOD}$ and a corresponding $3 \sigma$ noise level of $5.4 \mathrm{mOD}$. 


\section{Performance comparison with traditional metal-based SEIRA geometries}

We assess the performance of our dielectric metapixel design by numerically comparing it to a state-of-the-art SEIRA approach based on metallic nanostructures. Specifically, we follow the guidelines for periodicity optimized gold nanoantenna SEIRA substrates outlined in Ref. (20) to obtain resonant metallic metapixel designs in our target spectral range. The reflectance spectrum of the metallic metapixel displays a pronounced peak centered at $1560 \mathrm{~cm}^{-1}$ and a FWHM of $565 \mathrm{~cm}^{-1}$ (Fig S4.1A). The antenna geometry is defined as antenna length $2.1 \mu \mathrm{m}$, antenna width $0.14 \mu \mathrm{m}$, and antenna height $0.1 \mu \mathrm{m}$. The distance between antennas is $1 \mu \mathrm{m}$ in $\mathrm{x}$-direction and $2 \mu \mathrm{m}$ in y-direction.

To gauge the molecular fingerprint detection performance, the metallic metapixel surface is covered with a $2.5 \mathrm{~nm}$ thick conformal model protein layer analogous to the simulations for the dielectric metapixels presented in Fig. 1E of the main text. Even though the addition of the protein layer produces a clear change of the resonance lineshape $\left(R_{S}\right)$ compared to the reference spectrum $\left(\mathrm{R}_{0}\right)$, the protein-induced reflectance modulation is much lower compared to the dielectric metapixel case (Fig. S7A).

To quantify the performance difference between the metallic and dielectric metapixel designs, we calculate the absorbance signal from the reflectance curves $R_{0}$ and $R_{S}$ via $A=-\log \left(R_{S} / R_{0}\right)$. We find maximum absorbance signals for around $600 \mathrm{mOD}$ for dielectric case compared to around $40 \mathrm{mOD}$ for the metallic case, demonstrating that our dielectric metapixel concept can deliver performance improvements of more than one order of magnitude over state-of-the-art metal-based designs (Fig. S7B).
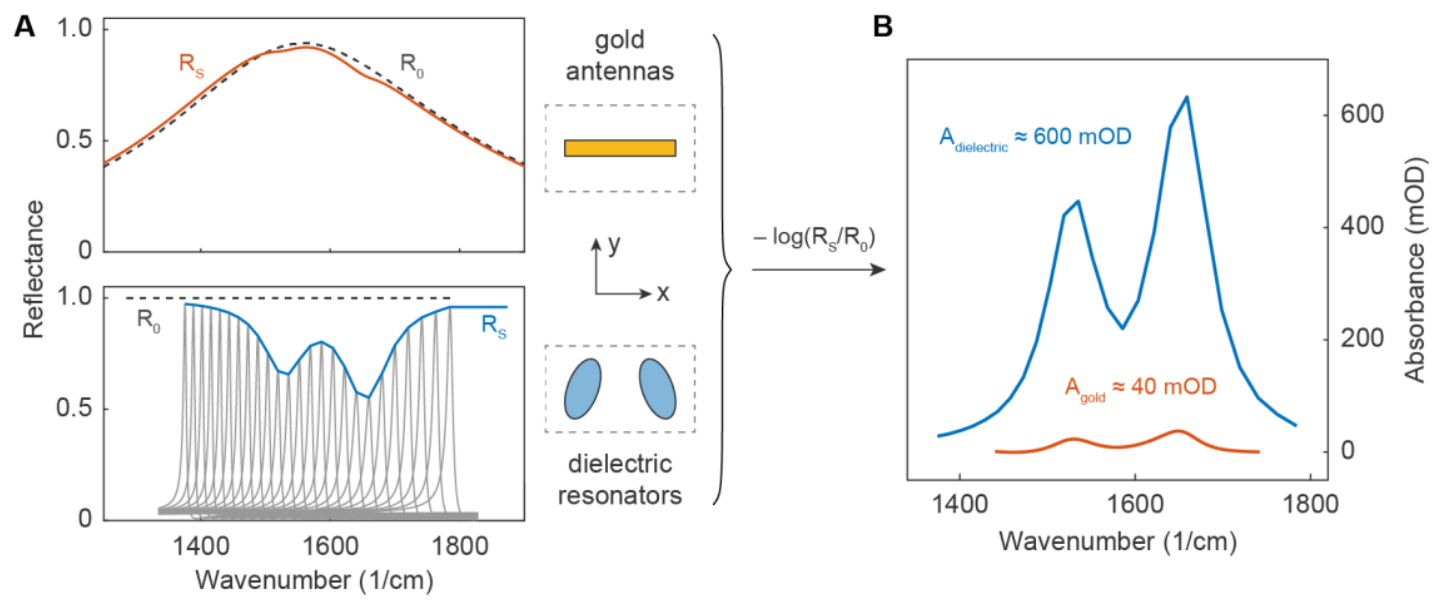

Figure S7: Molecular fingerprint detection performance comparison. (A) Simulated reflectance spectra for gold nanoantennas and our dielectric resonator concept before $\left(\mathrm{R}_{0}\right)$ and after $\left(\mathrm{R}_{\mathrm{S}}\right)$ addition of a conformal model protein layer. (B) Absorbance signal calculated from the reflectance curves $\mathrm{R}_{0}$ and $\mathrm{R}_{\mathrm{S}}$ from panel (A). The dielectric metapixel design provides an absorption signal improvement of over one order of magnitude. 
The key differentiating factor of our imaging-based dielectric metapixel concept is its ability to extract molecular fingerprints information from the integrated reflectance signal, which corresponds to the usage of a broadband light source and detector (see Fig. 3 in the main text). To check whether such performance is possible using metallic metapixels, we tune the length of the gold nanoantennas given above to produce a series of metallic metapixels with resonance frequencies covering the target fingerprint range (Fig. S8A). More specifically, the antenna length is linearly interpolated from $2.1 \mu \mathrm{m}$ to $3.1 \mu \mathrm{m}$ in 21 steps while keeping the other geometrical parameters the same.

Even though gold nanoantennas are chosen for the numerical calculations in accordance with literature, comparable performance is expected for other noble metals, which similarly act as near-perfect conductors at mid-infrared wavelengths.

A direct comparison between the metallic and dielectric metapixel cases is performed by integrating the reflectance spectra in the range from 1340 to $1840 \mathrm{~cm}^{-1}$ before $\left(\mathrm{I}_{0}\right)$ and after $\left(\mathrm{I}_{\mathrm{S}}\right)$ addition of the model protein layer. The integrated absorbance signal for both cases is then obtained via $\mathrm{A}=-\log \left(\mathrm{I}_{\mathrm{S}} / \mathrm{I}_{0}\right)$. We find that the integrated signal from the dielectric metapixels clearly reproduces the protein amide I and II absorption signature (Fig. S8B). In strong contrast, the integrated signal from the metallic metapixels is unable to resolve the protein signature, even though a slight variation of the reflectance envelope $R_{S}$ is still visible in Fig. S7A. Such comparatively poor performance is due to the broad linewidth of the gold nanoantenna resonances and the associated large integrated reflectance signal. Consequently, small proteininduced modulations of the reflectance signal have only a negligible influence on the total integrated signal, making broadband fingerprint detection with metallic metapixels challenging at realistic signal-to-noise levels.
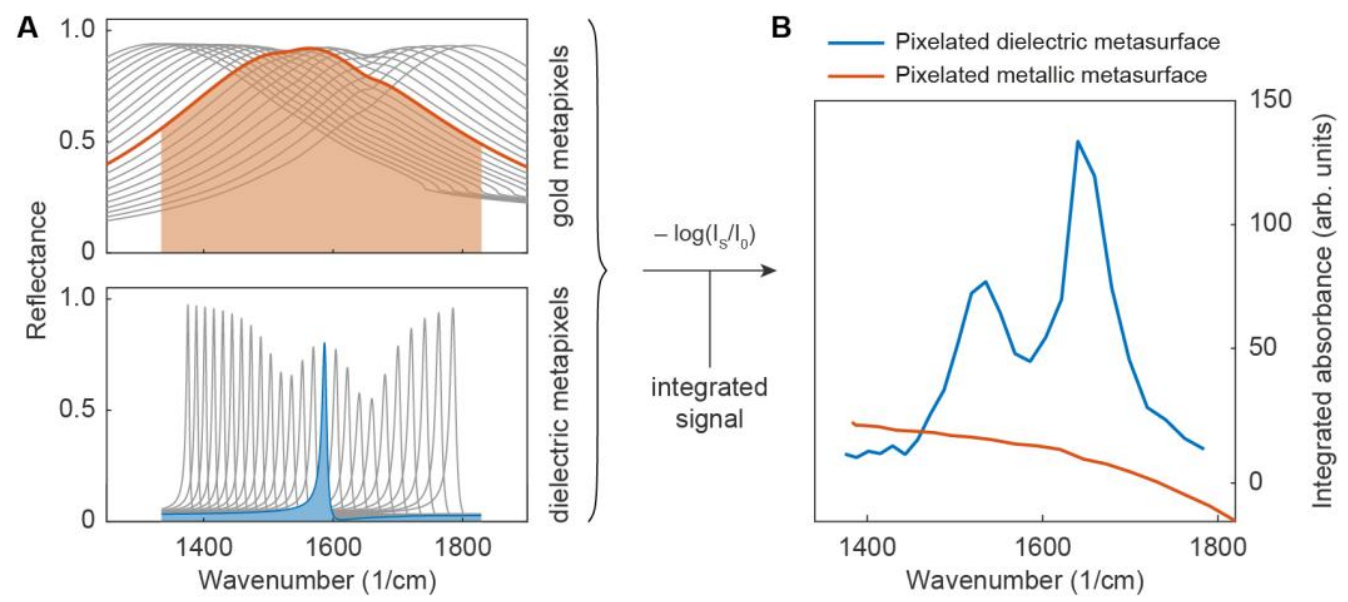

Figure S8: Imaging-based detection via integrated signals (A) Simulated reflectance spectra for gold and dielectric metapixels designed to exhibit resonances covering the amide I and II spectral range. (B) Integrated absorbance signal obtained by integrating the reflectance data in panel (A) from 1340 to $1840 \mathrm{~cm}^{-1}$. A pixelated metallic metasurface is unable to resolve the amide I and II absorption signature due to the high associated resonance linewidths. 


\section{Integration of sensor metasurface and imaging chip}

Our molecular fingerprint detection concept allows for a direct integration between sensor and imaging detector by placing the pixelated dielectric metasurface directly on a pixelated broadband IR detector such as a microbolometer or an array based on mercury cadmium telluride (MCT) elements (Fig. S9A). Such an integrated configuration requires the operation of the dielectric metasurface in transmission. In this case, the integrated absorbance from individual metapixels needs to be calculated via $A=-\log \left[\left(I_{S}-I_{B}\right) /\left(I_{0}-I_{B}\right)\right]$, where $I_{0}$ and $I_{S}$ denote the broadband readouts of the sensor metasurface before and after the addition of the analyte molecules, and $\mathrm{I}_{\mathrm{B}}$ represents the resonance-free background.

We perform molecular detection simulations similar to the ones in Fig. 1 of the manuscript with a design optimized for operation in transmission. This optimization yields geometrical parameters of $\mathrm{Px}=3.51 \mu \mathrm{m}, \mathrm{Py}=2.03 \mu \mathrm{m}$ for the unit cell periodicities, and $\mathrm{A}=1.76 \mu \mathrm{m}$, $\mathrm{B}=1.29 \mu \mathrm{m}$ for the ellipse long and short axes, with an orientation angle of $\theta=20^{\circ}$. Simulated transmittance spectra for scaling factors between $S=1.00$ and $S=1.36$ (linearly interpolated in 25 steps) are shown in Fig. S9B. Importantly, we find that the envelope of metapixel transmittance spectra still unambiguously reproduces the target molecular absorption fingerprint, with signal modulations up to $30 \%$.
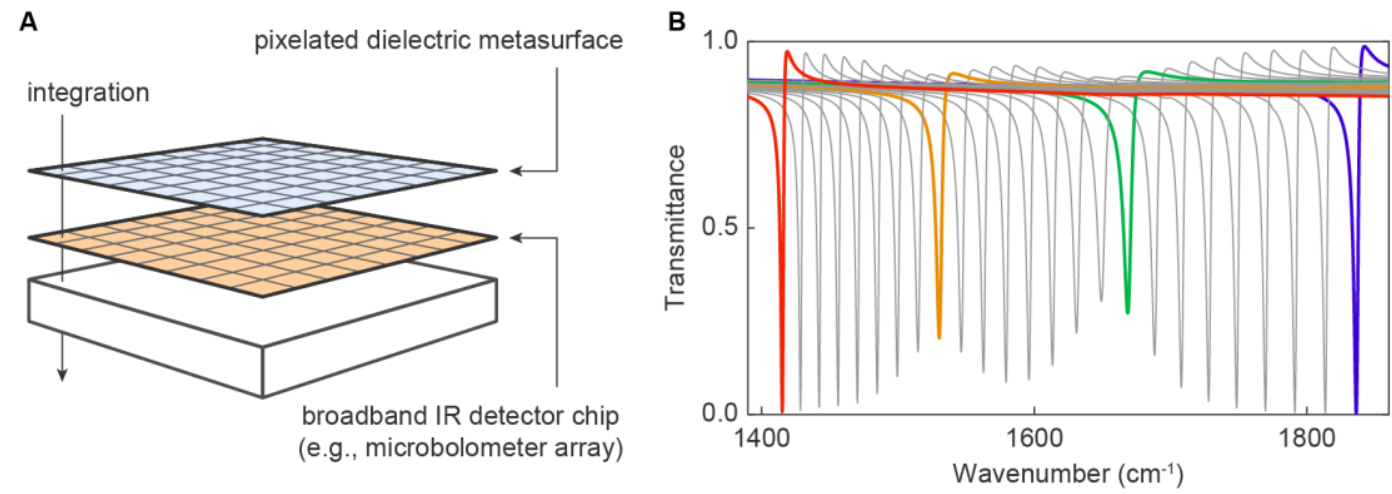

Figure S9: Towards chip-integration. (A) Sketch of a possible chip-integrated configuration for imaging-based molecular fingerprint detection. The pixelated metasurface sensor is placed directly on top of a pixelated broadband IR detector. (B) Fingerprint detection performance of the metasurface design in transmission. 